\title{
Evolving standards in preoperative staging and treatment of rectal cancer
}

\author{
Jensen TC Poon*, FCSHK, FHKAM (Surgery) \\ Division of Colorectal Surgery, Department of Surgery, The University of Hong Kong, Pokfulam, Hong Kong
}

Hong Kong Med J 2014;20:364-5

DOI: $10.12809 / \mathrm{hkmj} 144370$

Colorectal cancer has become the commonest cancer in Hong Kong since 2011 and rectal cancer constitutes about one third of all colorectal cancers. ${ }^{1}$ Rectal cancer has a much higher local recurrence rate of about $10 \%$ than colon cancer. ${ }^{2}$ Hence, stern efforts must be made to safeguard patients from recurrence during the management of rectal cancer. In current practice, good oncological outcome with low local recurrence rate for rectal cancer treatment relies on careful exercise of total mesorectal excision (TME) technique which is the standard for midand low-rectal cancer resection ${ }^{3}$ and perioperative radiotherapy with/without chemotherapy.

In the Dutch rectal cancer trial, combination of TME and preoperative short-course radiotherapy (5 Gy for 5 days) was associated with a significantly lower recurrence rate of $2.4 \%$ at 2 years versus $8.2 \%$ with TME only $(\mathrm{P}<0.001) .{ }^{4} \mathrm{~A}$ combination of longcourse radiotherapy (usually 50.4 Gy over 6 weeks) and fluorouracil offers additional benefit of tumour downstaging to improve sphincter preservation rate or even complete tumour remission in about $15 \%$ to $20 \%$ of patients. ${ }^{5}$ Radiotherapy, given after operation, can also reduce local recurrence rate. However, a randomised trial showed that, compared with postoperative chemoradiation, preoperative chemoradiation was associated with significantly better local control and less toxicity for locally advanced rectal cancer, which is defined as $\mathrm{T} 3$ or T4 or lymph node-positive rectal cancer. ${ }^{6}$ Hence, most colorectal centres adopt the policy of offering neoadjuvant (preoperative) chemoradiation to locally advanced rectal cancer.

As the preoperative local staging of rectal cancer affects the management plan, the accuracy of staging is very important. Preoperative local staging usually relies on endorectal ultrasound or magnetic resonance imaging (MRI). Recently, MRI has emerged as the preferred modality for local staging of rectal cancer by colorectal surgeons Apart from having high reproducibility and accuracy in assessing $\mathrm{T}$ stage and regional lymph node status, high-resolution MRI can predict circumferential resection margin (CRM) of the rectal tumour. In pathology terms, a positive CRM is defined as presence of tumour within $1 \mathrm{~mm}$ of radial surgical margin and it is associated with high chance of local recurrence. High-resolution MRI can accurately measure the closest distance between the tumour and mesorectal fascia and, hence, predict CRM. In a multicentre European trial (the MERCURY study), assessment of CRM by MRI was shown to be superior to TMN-based criteria in predicting local recurrence. After multivariate analysis, CRM was the only parameter that predicted local recurrence and patient survival in the preoperative stage. ${ }^{7}$ This finding suggested that MRI-predicted CRM assessment should be routinely incorporated into preoperative planning of rectal cancer treatment. The MERCURY study group proposed that the treatment plan of stage I to III rectal cancer can be guided by MRI assessment of rectal tumour. ${ }^{8}$ Good tumour prognosis by MRI is defined as predicted CRM of $>1 \mathrm{~mm}, \mathrm{~T} 1, \mathrm{~T} 2$ or T3 disease with depth of invasion of $<5 \mathrm{~mm}$ beyond muscularis propria $(\mathrm{T} 3 \mathrm{a} / \mathrm{b})$, irrespective of regional lymph node stage. Poor tumour prognosis by MRI is defined as predicted CRM of $<1 \mathrm{~mm}$ or T3 disease with depth of invasion of $>5 \mathrm{~mm}$ beyond muscularis propria $(\mathrm{T} 3 \mathrm{c} / \mathrm{d})$ or presence of extramural venous invasion. The centres involved in the MERCURY study had the policy of offering upfront surgery to tumours showing good prognosis by MRI and neoadjuvant chemoradiation to the tumours showing poor prognosis by MRI. The MERCURY study recorded local recurrence rate of only $3 \%$ in the tumours showing good prognosis by MRI and suggested omitting preoperative treatment in some stage III tumours. If the favourable results of the MERCURY study can be reproduced in other clinical trials, the MRI-predicted tumour prognosis system may become a new standard for deciding preoperative treatment of rectal cancer. However, the prerequisite for the success of a more selective approach in preoperative therapy is good TME technique by colorectal surgeons to avoid breaching of mesorectal fascia which may, otherwise, result in spillage of tumour cells and, subsequently, local recurrence. This is a serious concern when the tumour is covered and protected from exposure by only a few millimetres of CRM.

In the current issue of our journal, Wong et $\mathrm{al}^{9}$ report that the thickness of mesorectum in the Chinese is relatively thin and less than $15 \mathrm{~mm}$ in the majority of patients at most levels. As a result, the 
distance between the tumour and mesorectal fascia is intrinsically short. The CRM in Chinese patients with rectal cancer is reduced and, hence, more patients may have CRM which is involved or threatened by the tumour. This is a small series with only 25 patients and there is no similar report involving different ethnic groups for us to compare and ascertain if the mesorectum of the Chinese is thinner than that in patients of other ethnicities. However, we know that ultra-low rectal cancer (tumour within $5 \mathrm{~cm}$ from the anal verge) has a worse prognosis than higher tumour because the mesorectum tapers and thins out as it descends and approaches the pelvic floor. As a result, the chances of positive CRM and local recurrence increase. ${ }^{10}$ Neoadjuvant chemoradiation to downstage the tumour is particularly important in this group of patients with ultra-low tumours. It is also the policy of this author's centre to routinely offer neoadjuvant chemoradiation for ultra-low rectal cancer. Therefore, the hypothesis proposed by Wong et $\mathrm{al}^{9}$ is reasonable and underscores the importance of CRM during preoperative assessment.

The modern high-resolution MRI allows assessment of several characteristics of rectal cancer including the depth of tumour invasion, CRM, regional lymph node status, and extramural vascular invasion. These are features with prognostic value and serve to guide a more selective approach in neoadjuvant therapy of rectal cancer. In parallel with advances in chemoradiation and surgery, optimal care for rectal cancer is sophisticated and involves contribution from several specialists including radiologists, oncologists, pathologists, and colorectal surgeons. It is important that we keep abreast of advances in this field and manage patients within a multidisciplinary setting.

\section{References}

1. Hong Kong Cancer Registry, Hospital Authority. Summary of cancer statistics in Hong Kong in 2011. Available from: http://www3.ha.org.hk/cancereg. Accessed Aug 2014.

2. Poon JT, Law WL. Laparoscopic resection for rectal cancer: a review. Ann Surg Oncol 2009;16:3038-47.

3. Heald RJ, Moran BJ, Ryall RD, Sexton R, MacFarlane JK. Rectal cancer: the Basingstoke experience of total mesorectal excision, 1978-1997. Arch Surg 1998;133:894-9.

4. van Gijn W, Marijnen CA, Nagtegaal ID, et al. Preoperative radiotherapy combined with total mesorectal excision for resectable rectal cancer: 12-year follow-up of the multicentre, randomised controlled TME trial. Lancet Oncol 2011;12:575-82.

5. Maas M, Beets-Tan RG, Lambregts DM, et al. Waitand-see policy for clinical complete responders after chemoradiation for rectal cancer. J Clin Oncol 2011;29:4633-40

6. Sauer R, Becker H, Hohenberger W, et al. Preoperative versus postoperative chemoradiotherapy for rectal cancer. N Engl J Med 2004;351:1731-40.

7. Taylor FG, Quirke P, Heald RJ, et al. Preoperative magnetic resonance imaging assessment of circumferential resection margin predicts disease-free survival and local recurrence: 5-year follow-up results of the MERCURY study. J Clin Oncol 2014;32:34-43.

8. Taylor FG, Quirke P, Heald RJ, et al. Preoperative highresolution magnetic resonance imaging can identify good prognosis stage I, II, and III rectal cancer best managed by surgery alone: a prospective, multicenter, European study. Ann Surg 2011;253:711-9.

9. Wong EM, Lai BM, Fung VK, et al. Limitation of radiological T3 subclassification of rectal cancer due to paucity of mesorectal fat in Chinese patients. Hong Kong Med J 2014;20:366-70.

10. West NP, Finan PJ, Anderin C, Lindholm J, Holm T, Quirke P. Evidence of the oncologic superiority of cylindrical abdominoperineal excision for low rectal cancer. J Clin Oncol 2008;26:3517-22. 